\title{
Free-Electron-Driven Orbital Angular Momentum Emitter
}

\author{
Juan-Feng Zhu \\ State Key Laboratory of Advanced \\ Optical Communication Systems and \\ Networks \\ Department of Electronics, Peking \\ University \\ Beijing 100871, China
}

Fan-Hong Li

State Key Laboratory of Advanced

Optical Communication Systems and

Networks

Department of Electronics, Peking

University

Beijing 100871, China

Liang Zhang

Department of Physics, SUPA

University of Strathclyde

Glasgow G4 0NG, Scotland, UK

\author{
Chao-Hai \\ State Key Laboratory of Advanced \\ Optical Communication Systems and \\ Networks \\ Department of Electronics, Peking \\ University \\ Beijing 100871, China \\ *E-mail: duchaohai@pku.edu.cn \\ $\mathrm{Pu}-\mathrm{Kun} \mathrm{Liu}$ \\ State Key Laboratory of Advanced \\ Optical Communication Systems and \\ Networks \\ Department of Electronics, Peking \\ University \\ Beijing 100871, China
}

\author{
Zi-Wen Zhang \\ State Key Laboratory of Advanced \\ Optical Communication Systems and \\ Networks \\ Department of Electronics, Peking \\ University \\ Beijing 100871, China
}

\author{
Adrian W. Cross \\ Department of Physics, SUPA \\ University of Strathclyde \\ Glasgow G4 0NG, Scotland, UK
}

\begin{abstract}
A free-electron-driven orbital angular momentum (OAM) emitter is introduced in this paper. When the free electron passes over the cylindrical grating, the spoof surface plasmon (SSP) is stimulated in this system. With loading a helical wire which induces a gradient azimuthal phase distribution the in this system, the SSP mode will be converted into the spatial OAM radiation. The operating frequency of the OAM mode can be tuned by the electron beam energy of the surface plasmon. Compared with the Smith-Purcell radiation from the helical grating, the field intensity is enhanced by about 50 times. This method provides an efficient way to generate the OAM mode, and may find applications in the communication system.
\end{abstract}

\section{INTRODUCTION}

Angular momentum including spin angular momentum (SAM) and orbital angular momentum (OAM) has intrigued long-held interest in physical fields. SAM is related to the polarization of light and was experimentally verified in the 1930s [1]. Unlike SAM, the light carrying with the OAM is associated with beam vorticity and phase singularity, and was investigated in the 1990s [2-3]. Since then, OAM modes have been studied, and find many applications in various fields such as optical microscopy, micromanipulation, super-resolution imaging, communication system and so forth [4-5]. Owing to various applications, the approaches to generate EM waves carrying OAMs become a focused research. Up to now, there have been several ways to generate OAM mode. Initially, the spiral phase plate that can provide helical phase compensation to convert a Gaussian beam into an OAM mode was proposed. Similarly, the hologram plane capable of achieving the helical phase distribution and producing the OAM mode has also been proposed [6]. Besides, the metasurface, spoof surface plasmon (SSP) and antenna array can also be used to achieve the OAM mode [7-9].

However, despite many advanced progresses have been achieved, there are still some obstacles to be overcome. For instance, the radiation direction of the OAM mode cannot be manipulated. Encouragingly, the OAM mode can be implemented with the excitation of the free electron, which provides an efficient way to tailor the radiation direction of the OAM mode. Previous studies have shown that Smith-Purcell radiation is a promising way to achieve the OAM mode, and the radiation direction can be tuned by the energy of the free electron [10]. However, the radiation intensity is still weak. In the paper, the free-electron-driven OAM emitter based on the SSP is proposed. When the swift free electron flies through the cylindrical grating, the SSP mode is excited and converted into the radiation in the free space via the helical wire, which carries the OAM mode. The radiation is related to the period of the helical wire and the energy of the free electron. Compared with the conventional Smith-Purcell radiation form the helical grating, the radiation is enhanced about 50 times. This method can be utilized to generate the OAM mode in the terahertz $(\mathrm{THz})$ band.

\section{ANALYSIS AND RESULTS}

\section{A. Model Presentation and Dispersion Analysis}

The structure of the free-electron-driven OAM generator is shown in Fig. 1, which composes of a cylindrical grating and a helical grating. The period, width and radius of the cylindrical 
grating are $\mathrm{d}=0.1 \mathrm{~mm}, a=0.05 \mathrm{~mm}$ and $R_{1}=0.2 \mathrm{~mm}, R_{2}=$ $0.5 \mathrm{~mm}$. respectively. The period of the helical wire is $p=0.8$ $\mathrm{mm}$, and the radius of the wire is $R=0.1 \mathrm{~mm}$. The free electron bunch flies over the gap to excite the coherent $\mathrm{THz}$ radiation.

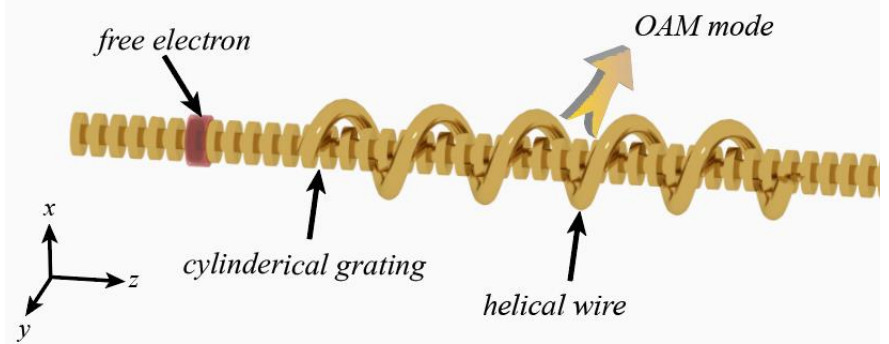

Fig. 1 The schematic model of the free-electron-driven OAM emitter. The system consists a cylindrical grating and a helical wire, and the free electron bunch flies through the gap between the grating and helical wire to excite the free $\mathrm{THz}$ radiation.

To investigate the physical mechanism about this system, the dispersion relation is analyzed firstly. As the metal behaves like a perfect electric conductor (PEC) in the $\mathrm{THz}$ range, the dispersion equation is derived according to the mode-matching method as Eq. (1).

$\sum_{n=-\infty}^{+\infty} S_{n}^{2} \frac{k_{0}}{k_{r n}} \frac{K_{1}\left(k_{r n} R\right)}{K_{0}\left(k_{r n} R_{2}\right)} \frac{N_{0}\left(k_{0} R_{2}\right) J_{0}\left(k_{0} R_{1}\right)-N_{0}\left(k_{0} R_{1}\right) J_{0}\left(k_{0} R_{2}\right)}{N_{0}\left(k_{0} R_{1}\right) J_{1}\left(k_{0} R_{2}\right)-N_{1}\left(k_{0} R_{2}\right) J_{0}\left(k_{0} R_{1}\right)}=1(1 \mathrm{a})$

$$
S_{n}=\sqrt{\frac{a}{d}} \operatorname{sinc}\left(\frac{\beta_{n} a}{2}\right)
$$

where, the $\beta_{n}=\beta_{0}+2 n \pi / d$ is the longitudinal wave vector of n-th harmonic wave, $k_{r n}=\sqrt{\mathrm{k}_{0}^{2}-\beta_{n}^{2}}, k_{0}=\frac{\omega}{c}=\frac{2 \pi f}{c}, \quad \mathrm{c}$ is the light speed in free space. Besides, the dispersion characters of the free electron beam are illustrated in Eq. (2)(3).

$$
\begin{gathered}
\omega=v_{e} \beta \\
v_{e}=c \sqrt{1-1 /\left(1+\frac{e U}{m_{0} c^{2}}\right)^{2}}
\end{gathered}
$$

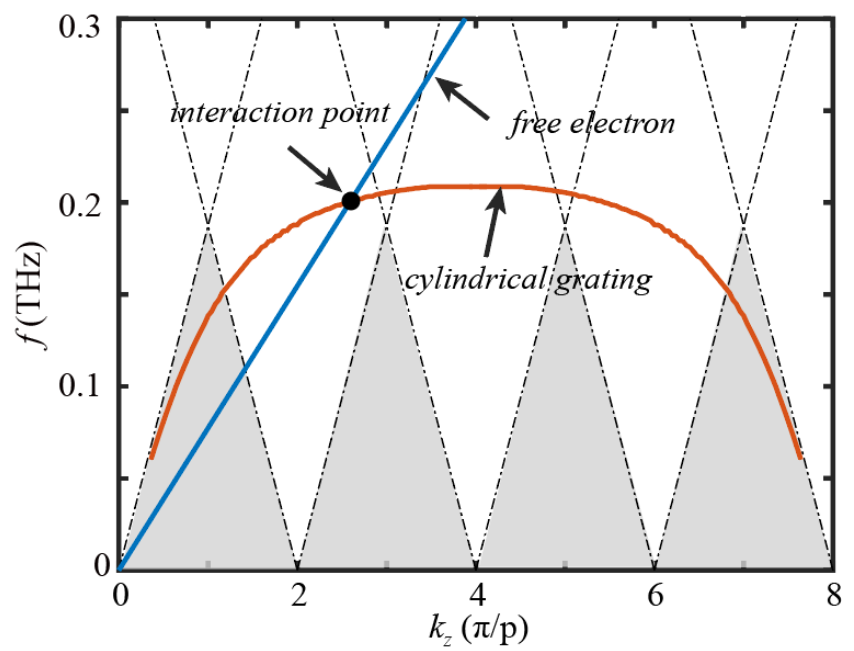

Fig. 2 The Brillouin diagram of the system. The dispersion curve of the cylindrical grating is marked in red, and the dispersion curve of the free electron is marked in blue.

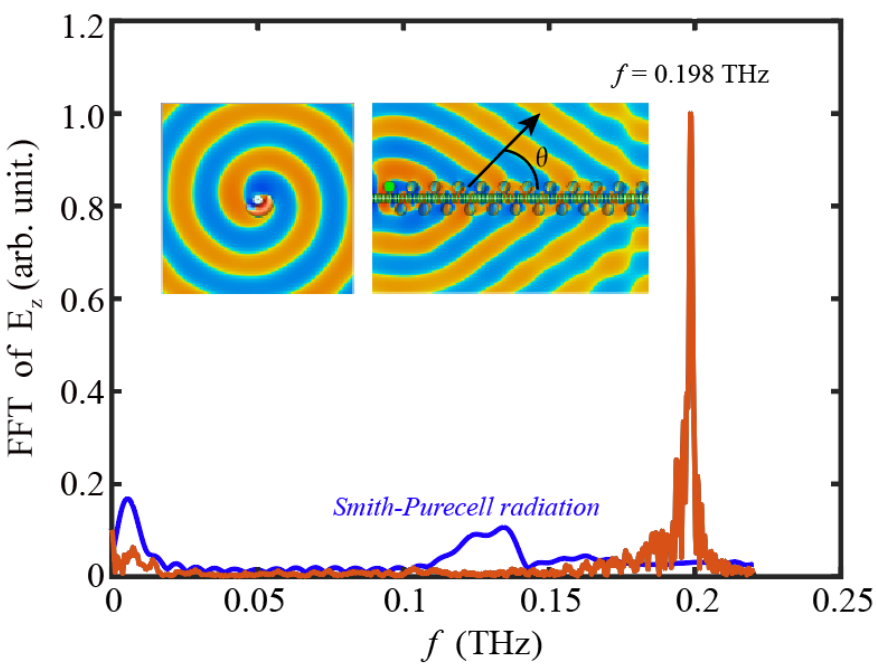

Fig. 3 Simulation results. The frequency spectrum probed at the far field. Inset: the transversal and longitudinal electric field profile at the radiation frequency

When the free electron beam passes over the cylindrical grating, the SSP mode can be excited. The radiation frequency is determined by the frequency of interaction point between the free electron and the dispersion curve, which is marked in Fig. 2. The helical wire induces a helical phase distribution in the transversal direction and converts the SSP mode into the OAM mode in free space. Besides, with the structural parameters remaining unchanged, the radiation frequency can be tuned by the electron beam voltage. The theoretical analysis is verified using the particle-in-cell (PIC) solver in the commercial software CST particle studio [11], and the simulation results are shown in Fig. 3. The frequency spectrum is probed in the far field and there is an obvious frequency peak at $f=0.198 \mathrm{THz}$, which agrees with the theoretical analysis. The corresponding electric field contour is depicted in the inset of Fig. 3, and the radiation angle is $70^{\circ}$, which is well agreed with the SmithPurcell radiation (SPR) dispersion relation.

$$
\lambda=\frac{p}{|n|}\left(\frac{c}{v_{e}}-\cos \theta\right)
$$

where $\lambda$ is the radiation wavelength, $\theta$ is the radiation angle with respect to the propagation direction of the electron bunch, $p$ is the diffraction period, and the integer $n$ is the order of diffraction, $v_{e}$ is the velocity of the free electron. In the system presented in this paper, $n=-1$.

Besides, the Smith-Purcell radiation with the helical wire is also shown in Fig. 3. The parameters remain unchanged. The lowest SPR frequency band based on the rod array ranges from $0.11 \mathrm{THz}$ to $0.26 \mathrm{THz}$, and the maximum field intensity of the SPR is about 0.02 in this frequency range. Compared with the ordinary SPR, the coherent $\mathrm{THz}$ radiation shows an enhancement more than 50 times, which implies that the radiation is promising for developing a compact and efficient radiation source.

\section{CONCLUSION}

A free-electron-driven OAM emitter is demonstrated in this paper. This emitter is consisted of a cylindrical grating and helical wire. The SSP on the cylindrical grating is excited by the free electron and transformed into the spatial OAM mode by the 
helical wire. The radiation frequency and radiation direction are tuned by the energy of free electron beam. Besides, the field intensity is about 50 times than that of ordinary SPR from the helical wire. The mechanism illustrated in this paper may open new avenues for generating OAM mode in the THz range.

\section{ACKNOWLEDGMENT}

This work was supported in part by the National Natural Science Foundation of China under contracts 61861130367, 61531002, NSAF-U1830201, and 61971013. It was also supported in part by the Newton Advanced Fellowship from Royal Society (NAF/R1/180121), United Kingdom.

\section{REFERENCES}

[1] A. Beth, "Mechanical detection and measurement of the angular momentum of light," Physical Review A: Atomic, Molecular and Optical Physics, vol. 50, no. 2, pp. 115-125, 1936.

[2] L. Allen, L. Allen, M. W. Beijersbergen, R. J. C. Spreeuw, and J. P. Woerdman, "Orbital angular momentumof light and the transformation of Laguerre-Gaussian laser modes," Physical Review A: Atomic, Molecular and Optical Physics, vol. 45, no. 11, pp. 8185 - 8189, 1992.

[3] H. He,M. E. J. Friese,N. R. Heckenberg, and H. Rubinsztein-Dunlop, "Direct observation of transfer of angular momentum to absorptive particles from a laser beam with a phase singularity," Physical Review Letters, vol. 75 , no. 5 , pp. 826-829, 1995

[4] Franke - Arnold S, Allen L, Padgett M. Advances in optical angular momentum Laser \& Photonics Reviews, vol.2, no. 4: 299-313, 2008.

[5] Padgett M, Bowman R. Tweezers with a twist. Nature photonics, vol. 5, no. 6, pp. 343-348, 2011.

[6] Shen Y, Wang X, Xie Z, et al. Optical vortices 30 years on: OAM manipulation from topological charge to multiple singularities. Light: Science \& Applications, vol.8, no. 1, pp 1-29, 2019

[7] Yin J Y, Ren J, Zhang L, et al. Microwave Vortex - Beam Emitter Based on Spoof Surface Plasmon Polaritons. Laser \& Photonics Reviews, vol.12, no.3, pp.1600316, 2018

[8] Yu S, Li L, Shi G, et al. Design, fabrication, and measurement of reflective metasurface for orbital angular momentum vortex wave in radio frequency domain. Applied Physics Letters, vol.108, no. 12, pp.121903, 2016.

[9] Mohammadi S M, Daldorff L K S, Bergman J E S, et al. Orbital angular momentum in radio-A system study. IEEE transactions on Antennas and Propagation, vol. 58, no. 2, pp. 565-572, 2009.

[10] Jing L, Wang Z, Lin X, et al. Spiral field generation in Smith-Purcell radiation by helical metagratings. Research, pp. 3806132, 2019.

[11] CST Corp., "CST PS Tutorials," http://www.cst-china.cn/. 